\title{
PREVENÇÃO DO TABAGISMO NO ESPAÇO ESCOLAR: RELATO DE EXPERIÊNCIA DE ESTUDANTES DE ENFERMAGEM
}

\author{
Maria Cristina Pinto de Jesus \\ Universidade Federal de Juiz de Fora \\ mariacristina.jesus@ufjf.edu.br \\ Lucélia Emily da Silva \\ Universidade Federal de Juiz de Fora \\ luceliaemely@yahoo.com.br \\ Davi dos Reis Alves \\ Universidade Federal de Juiz de Fora \\ davireis.alvesjf@gmail.com \\ Jéssica dos Santos Salgueiro \\ Universidade Federal de Juiz de Fora \\ jessicasalgueiro23@gmail.com
}

Pérola Furtado Barreto

Universidade Federal de Juiz de Fora perolafb@hotmail.com

Adriely de Abreu Varoto Universidade Federal de Juiz de Fora adrielyvarotto82@gmail.com

Ediane Mendes Lins Universidade Federal de Juiz de Fora edianejf@gmail.com

Maria Júlia Pereira de Souza Universidade Federal de Juiz de Fora mariajpsouza@hotmail.com.br

Fabiana de Oliveira Freitas Centro de Ensino Superior de Juiz de Fora fabianaoliveiraf@yahoo.com.br

\section{Resumo}

Objetivo: Relatar a realização de ações de prevenção do hábito de fumar cigarros, voltadas a adolescentes no espaço escolar, na modalidade de extensão universitária. Método: Relato de experiência de ações extensionistas com adolescentes entre 11 e 19 anos de uma escola pública, realizadas de novembro de 2018 a junho de 2019. Resultados: A maioria dos participantes demonstrou conhecimento sobre tabagismo e substâncias nocivas do cigarro, especialmente a nicotina; associou o tabagismo com doenças cardiopulmonares, cânceres e demonstrou conhecer a importância da prevenção desse hábito para evitar prejuízos à saúde e ao meio ambiente; bem como apontou a importância de manter hábitos saudáveis, obter informações sobre tabagismo, não ser influenciada e ficar distante de fumantes como forma de prevenção. Conclusão: Para os adolescentes, o projeto contribuiu para o aprendizado e para o compartilhamento deste com outras pessoas. A experiência foi considerada relevante para a formação profissional dos acadêmicos de enfermagem envolvidos. Palavras-chave: Prevenção do Hábito de Fumar. Educação em Saúde. Adolescente.

\section{SMOKING PREVENTION IN SCHOOL SPACE: REPORT CASE OF NURSING STUDENTS}

\section{Abstract}

Objective: To report on cigarette smoking prevention activities with adolescents in the school space in the university extension modality. Method: Report of experience of extension actions with adolescents between 11 and 19 years of age in a public school, carried out from November 2018 to June 2019. Results: Most participants showed knowledge about smoking and harmful substances in cigarettes, especially nicotine. They associated smoking with cardiopulmonary diseases, cancers and demonstrated to know the importance of preventing this habit to avoid health and environmental problems. They pointed out the importance of maintaining healthy habits, obtaining information about smoking, not being influenced and staying away from smokers as a form of prevention. Conclusion: For the adolescents, the project contributed to their learning, as well as the possibility of sharing it with others. The experience was considered relevant for the professional education of the nursing students involved.

Keywords: Smoking Prevention. Health Education. Adolescent. 


\title{
PREVENCIÓN DE FUMAR EN EL ESPACIO ESCOLAR: RELATO DE EXPERIENCIA DE ESTUDIANTES DE ENFERMERÍA
}

\begin{abstract}
Resumen
Objetivo: Informar sobre las actividades de prevención del tabaquismo con adolescentes en el espacio escolar en la modalidad de extensión universitaria. Método: Informe de experiencia de acciones de extensión con adolescentes entre 11 y 19 años en una escuela pública, realizada desde noviembre de 2018 hasta junio de 2019. Resultados: La mayoría de los participantes mostraron conocimiento sobre fumar y sustancias nocivas en los cigarrillos, especialmente la nicotina. Asociaron fumar con enfermedades cardiopulmonares, cánceres y demostraron conocer la importancia de prevenir este hábito para evitar problemas de salud y ambientales. Señalaron la importancia de mantener hábitos saludables, obtener información sobre fumar, no ser influenciado y mantenerse alejado de los fumadores como una forma de prevención. Conclusión: Para los adolescentes, el proyecto contribuyó a su aprendizaje, así como a la posibilidad de compartirlo con otros. La experiencia se consideró relevante para la educación profesional de los estudiantes de enfermería involucrados.

Palabras clave: Prevención del Hábito de Fumar. Educación en Salud. Adolescente.
\end{abstract}




\section{INTRODUÇÃO}

A adolescência pode ser exemplificada como uma construção biopsicossocial que carrega consigo a estruturação tanto da imagem corporal, quanto da personalidade. Essa fase pode incluir situações que merecem a atenção dos serviços públicos com foco nas vulnerabilidades, tais como gravidez não planejada, abuso de álcool e outras drogas (FERREIRA et al., 2016).

A educação em saúde é uma importante ferramenta para a atuação em enfermagem com enfoque na promoção de saúde nos mais diversos ambientes. A Declaração de Ottawa estabelece que o processo de educação em saúde deve estar voltado para as necessidades e demandas da comunidade, fazendo com que os indivíduos sejam agentes importantes no processo de educação (FEIO; OLIVEIRA, 2015).

A escola representa um espaço estratégico para atuação do enfermeiro com adolescentes no contexto da Atenção Primária à Saúde (APS), especialmente na realização de atividades de promoção da saúde (BRASIL, 2017). Por isso, a atuação em escolas e outros equipamentos sociais deve fazer parte da rotina dos profissionais de enfermagem das Unidades Básicas de Saúde (UBSs).

A revisão integrativa que analisou o processo de trabalho do enfermeiro na APS concluiu que, apesar das dificuldades, este profissional é reconhecido como detentor de características essenciais à visão ampliada do processo saúde-doença, sendo apontado como apto para aproximar as políticas públicas da equipe e da comunidade, planejar as atividades de saúde, assim como coordenar ações e serviços nesse nível de atenção (DUTRA et al., 2016).

A prevenção do tabagismo, considerado um importante problema de saúde pública e principal causa evitável de morbidade e mortalidade prematura, é uma das ações de promoção da saúde que o enfermeiro pode desenvolver no âmbito da APS. Salienta-se que o uso do tabaco matou 100 milhões de pessoas no século passado, podendo chegar a 1 bilhão no século XXI (WHO, 2015).

O tabagismo na população jovem associa-se a um significativo aumento de problemas de saúde durante a infância e a adolescência, além de se configurar como um importante fator de risco para doenças crônicas não transmissíveis, como cânceres, diabetes mellitus, doenças cardiovasculares e respiratórias (OLIVEIRA-CAMPOS et al., 2018).

A literatura mostra que os adolescentes constituem uma parcela da população vulnerável ao hábito de fumar. Os dados da National Youth Tobacco Surveys (NYTS) aplicada aos escolares entre os anos de 2011 e 2014, nos Estados Unidos da América, ressaltaram que o cigarro eletrônico foi o produto de tabaco mais frequentemente utilizado pelos estudantes $(9,4 \%)$, tanto do Ensino Médio quanto do Ensino Fundamental, seguido do narguilé (2,5\%). O maior 
crescimento da prevalência de uso desses produtos do tabaco aconteceu entre 2013 e 2014. Especificamente em 2014, esses dados apontaram que 24,6\% dos estudantes norte-americanos que frequentavam o Ensino Médio fizeram uso de algum produto do tabaco (ARRAZOLA et al., 2015). No Peru, um estudo mostrou que a maioria dos adolescentes iniciou o uso do tabaco aos 14 anos, motivados pela curiosidade e pelos amigos, sem o conhecimento de seus pais. Os adolescentes homens tiveram uma maior frequência de consumo (VALLADARES-GARRIDO $e t$ al., 2017).

Estudo transversal com 109.104 escolares que cursaram o $9^{\circ}$ ano do Ensino Fundamental no Brasil mostrou que 4,8\% fizeram uso de produtos de tabaco. Os fatores que aumentaram a chance de uso do tabaco foram, entre outros: ser do sexo masculino, trabalhar, perceber que os pais ou responsáveis se importariam pouco caso eles fumassem, possuir pais ou responsáveis fumantes e ter presenciado pessoas fumando. Concluiu-se que a prevalência de consumo de outros produtos do tabaco é elevada entre estudantes brasileiros e está associada com melhores condições socioeconômicas, presença de comportamentos de risco e convívio em ambiente permissível ao consumo do tabaco (HALLAL et al., 2017). Nesse contexto, ressalta-se a importância de ações de promoção da saúde e prevenção do tabagismo entre adolescentes em escolas.

O efeito significativo de mudança da percepção dos adolescentes e dos educadores sobre tabagismo foi constatado em estudo realizado na Dinamarca. Ao se implementar um currículo com estratégias de estímulo à discussão sobre as consequências do tabagismo e ao se criar ambiente livre de fumo na escola, constatou-se redução do número de adolescentes fumantes, maior chance de o assunto ser discutido pelos professores em sala de aula, além de maior diálogo dos estudantes em casa com a família sobre o assunto (BAST et al., 2016).

No Brasil, a Política Nacional de Controle do Tabagismo (PNCT), que tem entre suas diretrizes a "Escola Livre do Tabaco", envolve um conjunto de ações educativas, normativas e organizacionais que visam estimular mudanças de comportamento relacionadas ao tabagismo entre professores, estudantes e toda a comunidade que interage com a escola (BRASIL, 2015).

Diante do exposto, objetivou-se relatar a realização de ações voltadas a adolescentes, no espaço escolar, de prevenção do início do hábito de fumar cigarros, na modalidade de extensão universitária. 


\section{MATERIAIS E MÉTODOS}

Trata-se do relato de experiência de ações extensionistas sobre a prevenção do tabagismo entre adolescentes em uma escola pública, realizadas de novembro de 2018 a junho de 2019, por uma bolsista de extensão e seis voluntários, estudantes do Curso de Graduação em Enfermagem de uma universidade federal de um município de Minas Gerais, sob supervisão docente. A pesquisa-ação fundamentou o desenvolvimento das atividades de educação em saúde. Esse referencial teórico-metodológico objetiva obter experiências, produzir conhecimento e contribuir para a discussão sobre determinado tema, utilizando-se de seu caráter participativo, reflexivo e dialógico (THIOLLENT, 2009).

Antes de iniciar o trabalho educativo, foi realizado na escola um evento científico de sensibilização dos pais e responsáveis acerca da relevância da prevenção do uso do tabaco entre os adolescentes, intitulado "Importância do Ambiente sem Tabaco". O público-alvo foi constituído de adolescentes matriculados do $7^{\circ}$. ao $9^{\circ}$. ano do Ensino Fundamental de uma escola pública, com idades entre 11 e 19 anos, homens e mulheres, fumantes ou não.

Para abordagem dos participantes, foram utilizados recursos pedagógicos participativos, de modo a promover o envolvimento de todos, motivando a discussão do tema. Elaboraram-se banners com imagens sobre o tabaco, danos causados pelo cigarro ao meio ambiente e à saúde, além da importância de hábitos saudáveis de vida na infância e na adolescência.

A atividade educativa foi realizada em sala de aula e precedida pelo preenchimento por adolescentes de um formulário com perguntas pré-exposição ao tema (dependência do cigarro, impactos no meio ambiente) e sobre tabagismo (significado do termo, motivos que levam a fumar, problemas que provoca à saúde, meios de prevenção do uso do cigarro).

As intervenções tiveram duração média de 50 minutos e contaram com até 20 participantes em cada turma; ocorreram durante o horário escolar, em momento estabelecido com a direção da escola, considerando a disponibilidade dos professores para participar com a equipe de extensão. As seguintes questões nortearam a atividade educativa: o que o adolescente sabe sobre as consequências do hábito de fumar para a saúde e o meio ambiente? Que estratégias podem ser utilizadas para prevenir o hábito de fumar?

Ao final de cada intervenção, foi destinado um tempo para os adolescentes preencherem o formulário pós-exposição ao tema, contendo as mesmas questões respondidas por eles no início da atividade, com a finalidade de avaliar o impacto da discussão. Além da obtenção de dados dos formulários pré e pós-exposição ao tema, entrevistaram-se, em sala privativa na escola, 41 adolescentes com representação do $7^{\circ}$. ao $9^{\circ}$. ano do ensino fundamental, que se colocaram como voluntários para ceder depoimentos sobre o trabalho educativo. Para tal, utilizou-se a 
entrevista individual com questões abertas: fale o que mais chamou sua atenção durante a ação educativa. Você sabe dizer quais são os efeitos do fumo em sua saúde e no meio ambiente? O que você aprendeu sobre como prevenir o início do hábito de fumar? Essas entrevistas foram realizadas pela equipe de acadêmicos que foram treinados para entrevistar, gravar o áudio em smartphone e transcrever os depoimentos. Também os acadêmicos de enfermagem foram convidados a relatar seus pontos de vista sobre a atividade educativa, de modo a qualificar o relato de experiência.

Os dados numéricos foram digitados, organizados em uma planilha eletrônica, tratados descritivamente e apresentados em gráficos. Para a abordagem qualitativa, adotaram-se as fases: exploratória, que é definida pela observação do problema; de desenvolvimento, constituída pelo trabalho participativo, e de conclusão, em que é realizada a análise do conteúdo obtido, discussão e divulgação dos resultados (THIOLLENT, 2009). Dos depoimentos dos adolescentes foram extraídas unidades de significado que convergiam com o registro dos momentos de interação com os acadêmicos. De posse dos dados, centrou-se na compreensão do sentido das atividades educativas realizadas, segundo o ponto de vista dos participantes.

Para garantir o anonimato dos participantes, os trechos de depoimentos mencionados no texto foram identificados entre parênteses com a palavra "Adolescente", seguida de numeração arábica, atribuída após a transcrição das entrevistas. O projeto foi aprovado pelo Comitê de Ética em Pesquisa com seres humanos da universidade federal responsável pela ação extensionista, sob o Parecer no 2.984.843, de 26 de outubro de 2018. O Termo de Consentimento Livre Esclarecido foi assinado pelo professor responsável pela turma e o Termo de Assentimento, pelos adolescentes.

\section{RESULTADOS E ANÁLISES}

As intervenções educativas foram realizadas em sala de aula, envolveram 14 turmas e contaram com a presença do professor da disciplina. Participaram 138 adolescentes, de ambos os sexos, sendo 98 com idades entre 11 e 14 anos ( $7^{\circ}$. ao $8^{\circ}$. ano) e 40 entre 15 e 19 anos ( $9^{\circ}$. ano). Considerando as respostas pré e pós-exposição ao tema, constata-se que o quantitativo de 80 adolescentes $(57,9 \%)$ demonstrou saber o que é tabagismo e que a nicotina causa dependência. Observa-se no Gráfico 1 que, após a ação educativa realizada pelos acadêmicos de enfermagem, houve melhora desse conhecimento. 
Gráfico 1 - Conhecimento de adolescentes sobre o que é tabagismo e qual substância causa dependência do tabaco

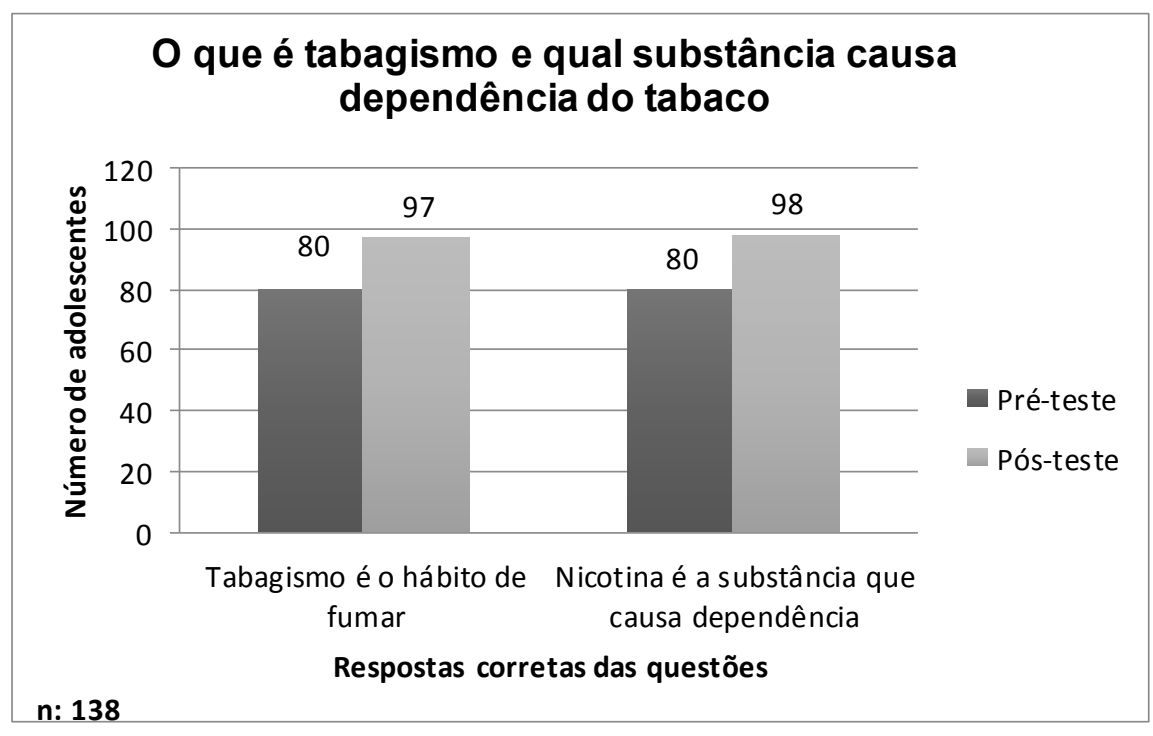

Fonte: elaborado pelos autores

Especialmente na adolescência, o ambiente escolar deve ser utilizado para influenciar o comportamento nessa etapa da vida com intervenções profissionais, visando à promoção da saúde e prevenção de agravos (BRASIL, 2017). Para promover saúde, é necessário valorizar a autonomia dos sujeitos em relação ao cuidado consigo mesmo e estimular a participação destes nas decisões que os afetam (MACEDO; CONCEIÇÃO, 2015). Além disso, profissionais de saúde e educadores, assim como pais e responsáveis, possuem função imprescindível na formação do pensamento crítico acerca da construção de hábitos saudáveis de vida, por meio de aconselhamento e momentos de diálogo continuamente com os adolescentes (VIERO et al., 2015).

O Gráfico 2 mostra as respostas de adolescentes sobre os problemas que o cigarro pode causar à saúde e ao meio ambiente. Evidenciou-se uma maior associação entre tabagismo e doenças cardiopulmonares, bronquite e cânceres e entre tabagismo e danos ao solo e saúde dos agricultores. 
Gráfico 2- Conhecimento de adolescentes sobre quais problemas o cigarro pode causar à saúde e ao meio ambiente

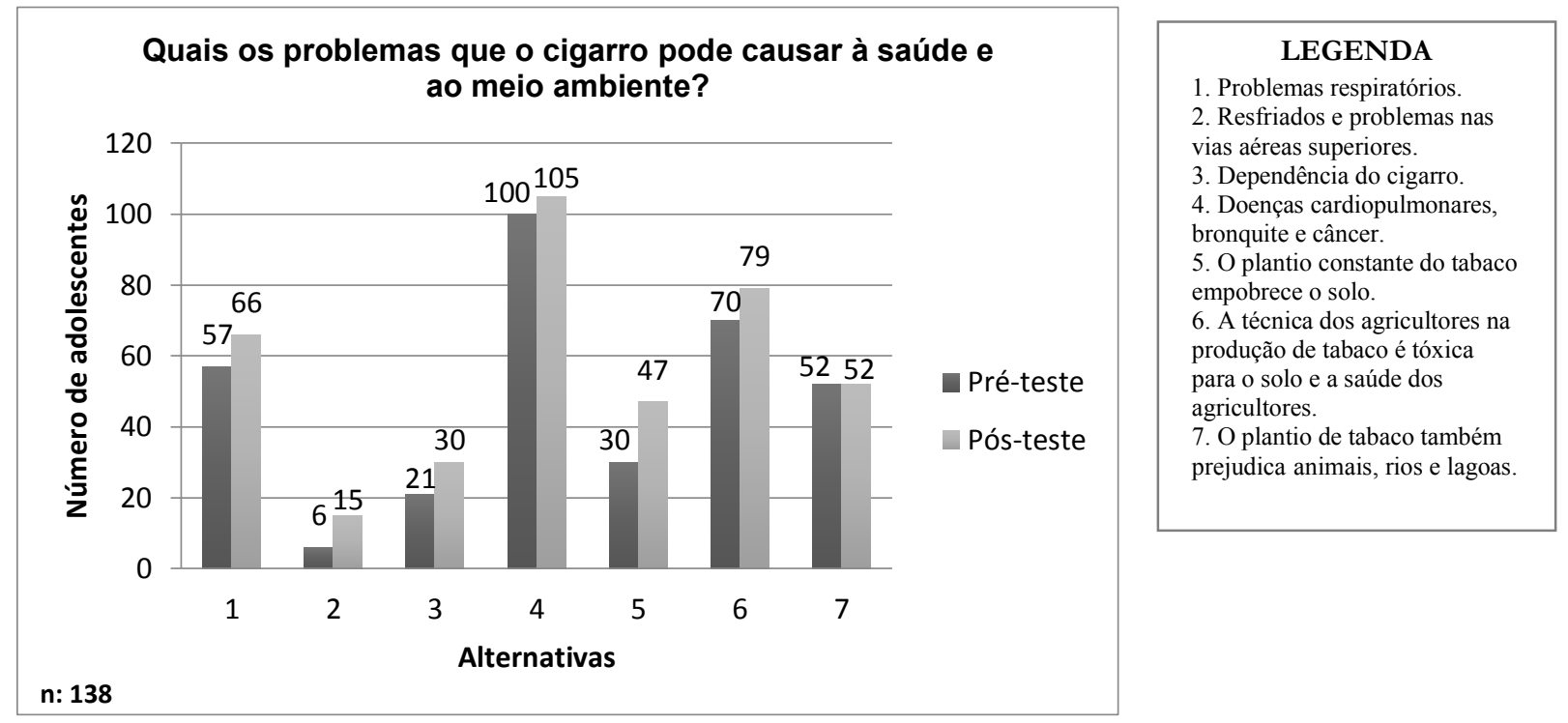

Fonte: elaborado pelos autores

Entre os problemas causados à saúde apontados pelos participantes como decorrentes do cigarro, as respostas que se sobressaíram foram as que relacionavam o tabagismo com doenças cardiovasculares, bronquite e câncer. As questões referentes ao meio ambiente ficaram mais evidentes para os adolescentes no momento do registro pós-exposição ao tema. Salienta-se que, durante a ação educativa, foram abordadas as questões sobre os danos ao solo, aos rios, às lagoas e à saúde dos agricultores e trabalhadores envolvidos na produção do tabaco.

A exposição ao tabaco na adolescência está associada ao desenvolvimento de algumas doenças crônicas não transmissíveis (doenças cardiovasculares, respiratórias e câncer). Nessa fase, existe maior vulnerabilidade ao fumo devido à curiosidade em explorar o novo (MALTA et al., 2014; PEREIRA et al., 2017). Nesse sentido, a iniciação precoce do uso do tabaco é um importante fator prognóstico para o adoecimento no futuro. Quanto mais cedo se estabelece a dependência ao tabaco, maior o risco de morte prematura na meia-idade ou na idade madura (BRASIL, 2015). As atividades de promoção da saúde tornam-se um importante instrumento de conscientização dos adolescentes quanto aos riscos do uso do tabaco.

Em uma questão de múltipla escolha em que se interrogava como se previne o início do uso do cigarro, as alternativas que mais chamaram a atenção dos adolescentes foram as que mencionavam hábitos saudáveis, como prática de exercícios físicos, esportes e manutenção de uma alimentação saudável, rica em nutrientes. O Gráfico 3 mostra as respostas dos adolescentes sobre as estratégias utilizadas para prevenir o início do uso do cigarro. 
Gráfico 3- Conhecimento dos adolescentes sobre como prevenir o uso do cigarro.

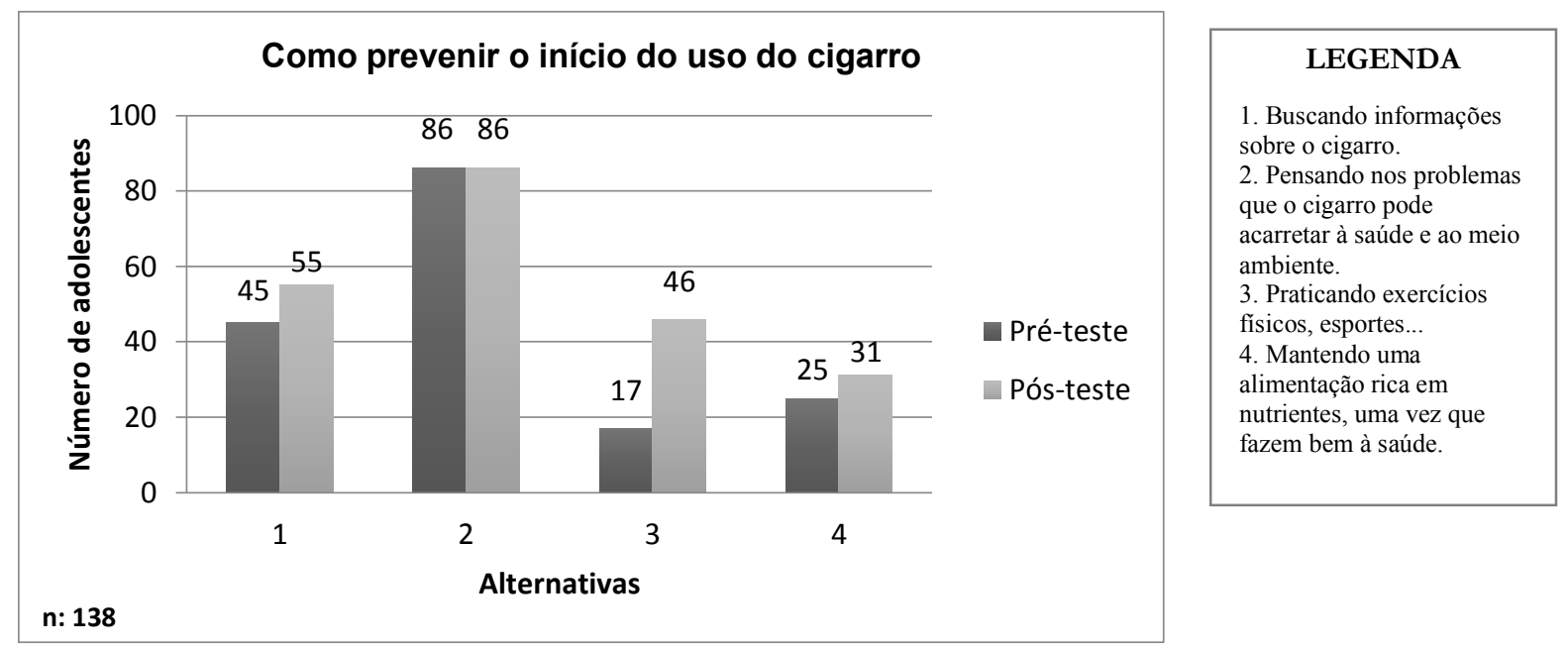

Fonte: elaborado pelos autores

Observa-se que não houve alteração nas respostas de antes e depois da exposição, no que diz respeito aos males que o cigarro pode fazer à saúde e ao meio ambiente. No entanto, constata-se que o conhecimento em relação à busca de informações sobre o cigarro, à importância da prática de exercícios e esportes, assim como de uma alimentação saudável, foi ampliado com a intervenção extensionista.

Considerando a vulnerabilidade de crianças e adolescentes ao uso de tabaco e outras drogas, a atuação do enfermeiro na escola poderá propiciar ações de promoção da saúde, com vistas ao enfrentamento das vulnerabilidades que comprometem o pleno desenvolvimento de crianças e jovens da rede pública de ensino (BRASIL, 2015).

Uma revisão sistemática apontou que intervenções comportamentais direcionadas a crianças e jovens em idade escolar podem prevenir o tabagismo e ajudar na cessação do tabaco. A metanálise mostrou que os participantes dessas intervenções apresentaram 18\% menos probabilidade de ter iniciado o tabagismo no final da intervenção, em relação ao grupo controle. Concluiu ser necessário incluir um acompanhamento em longo prazo (PEIRSON et al., 2016).

Alguns depoimentos de adolescentes ratificaram a importância das ações educativas, visando à prevenção do hábito de fumar. As falas sobre o que mais chamou atenção durante a ação educativa destacaram não somente que fumar é prejudicial à saúde como ao meio ambiente e solo, como também que é importante compartilhar as informações com outras pessoas:

"Eu não sabia que o cigarro causava esses tipos de doenças, que demorava muito para se decompor na natureza, que causava muitos danos na saúde". (Adolescente 1) 
"Meu pai podia parar de fumar e não jogar cigarro no chão, porque demora para se decompor". (Adolescente 7)

"[...] tem coisa que en não sabia, que agora en sei e que en posso compartilhar com as pessoas". (Adolescente 11)

Sobre o que os adolescentes aprenderam acerca de como prevenir o início do hábito de fumar, a maioria mencionou a importância de ter informações sobre as consequências do uso do cigarro, assim como de praticar exercícios físicos e manter uma dieta saudável. Os adolescentes salientaram a importância de não ser influenciados por fumantes:

'[...] primeiro, a gente deve saber o mal que o cigarro faz para gente... [...] fazer esportes, correr, caminhar, pelo menos 10 a 20 minutos...”. (Adolescente 25)

"[...] ter boa alimentação e ser saudável”. "Comida saudável e não ir pela cabeça dos outros". (Adolescente 14)

"Sair de perto das pessoas que fumam porque elas vão te influenciar... para ter menos vontade de querer fumar". (Adolescente 31)

Segundo os acadêmicos de enfermagem, as ações extensionistas oportunizaram o aprofundamento da relação entre profissionais de saúde e educação, bem como entre os demais acadêmicos e os adolescentes. Eles referiram a possibilidade de desenvolvimento de habilidades para a realização de atividades de promoção da saúde e prevenção de agravos na adolescência, como o tabagismo, por meio da vinculação entre a teoria e a prática no contexto da formação do enfermeiro.

Salienta-se a relevância de a equipe de saúde, durante os contatos rotineiros com crianças e adolescentes nos serviços de APS, em especial nos equipamentos sociais como a escola, oferecer informações sobre o hábito de fumar, aconselhando sobre os malefícios desse hábito, com foco na prevenção da iniciação tabágica. Essas informações podem ser realizadas por meio de oficinas, dinâmicas e palestras lúdicas acerca do tabagismo (BRASIL, 2015).

Como fator dificultador das atividades extensionistas apontam-se os períodos de paralisação de professores da rede municipal de ensino fundamental e médio, que provocaram descontinuidade das ações, contudo se reitera a importância de esses professores serem apoiados por profissionais de saúde das UBSs na realização de atividades voltadas para a promoção da saúde e prevenção do tabagismo na infância e adolescência. 


\section{CONSIDERAÇÕES FINAIS}

Considera-se que o objetivo de realizar, com adolescentes no espaço escolar, ações de prevenção do início do hábito de fumar cigarros, na modalidade de extensão universitária, foi atingido na medida em que, segundo os adolescentes, a intervenção educativa oportunizou o aprendizado sobre os malefícios do tabaco. Isso os ajuda a não iniciar o uso ou a parar de fumar, além de possibilitar a eles o compartilhamento desse aprendizado com outras pessoas, inclusive com seus familiares. Por outro lado, os estudantes de enfermagem consideraram que essa experiência extensionista ampliou as oportunidades de aprendizado sobre a prevenção do tabagismo na adolescência, qualificando a formação profissional. Além disso, salienta-se a contribuição da universidade no cumprimento de seu papel social ao estender sua relação com a comunidade, divulgando e compartilhando o conhecimento científico.

Desse modo, sinaliza-se a importância da continuidade da referida atividade extensionista para além do âmbito escolar, envolvendo as famílias dos adolescentes com vistas a potencializar seu alcance e beneficiar os envolvidos.

\section{REFERÊNCIAS}

ARRAZOLA R. A. et al. Tobacco Use Among Middle and High School Students - United States, 2011-2014. Morbidity and Mortality Weekly Report, v. 64, n. 14, p.381-5, 2015. Disponível em: https://www.cdc.gov/mmwr/preview/mmwrhtml/mm6414a3.htm. Acesso em: 25 maio. 2020.

BAST, L. S. et al. High impact of implementation on school-based smoking prevention: the X:IT study - a cluster-randomized smoking prevention trial. Implement Science, v. 11, n. 125, p.114, 2016.2 Disponível em: https://www.ncbi.nlm.nih.gov/pmc/articles/PMC5027074/pdf/13012_2016_Article_490.pdf. Acesso em: 25 maio. 2020.

BRASIL. Ministério da Saúde, Secretaria de Atenção à Saúde, Departamento de Ações Programáticas e Estratégicas. Proteger e cuidar da saúde de adolescentes na Atenção Básica. Brasília, 2017. Disponível em: http://bvsms.saude.gov.br/bvs/publicacoes/proteger_cuidar_adolescentes_atencao_basica.pdf. Acesso em: 25 maio. 2020.

BRASIL. Ministério da Saúde, Secretaria de Atenção à Saúde, Departamento de Atenção Básica. Estratégias para o cuidado da pessoa com doença crônica: o cuidado da pessoa tabagista. Brasília, 2015.2 Disponível em: http://189.28.128.100/dab/docs/portaldab/publicacoes/caderno_40.pdf. Acesso em: 25 maio. 2020. 
DUTRA, Carla Dias et al. Nursing working process in primary health care: integrative review. Revista de Enfermagem UFPE On Line, v.10(Supl. 3), p.1523-34, 2016. Disponível em: https://periodicos.ufpe.br/revistas/revistaenfermagem/article/view/11094/12549. Acesso em: 25 maio. 2020.

FEIO, Ana; OLIVEIRA, Clara Costa. Confluências e divergências conceituais em educação em saúde. Saúde e Sociedade, v. 24, n. 2, p.703-715, 2015. Disponível em http://www.scielo.br/pdf/sausoc/v24n2/0104-1290-sausoc-24-02-00703.pdf. Acesso em 25 maio. 2020.

FERREIRA, C. P. S. et al. Educational strategies for health education with teens: a review integrative. Revista Online de Pesquisa: Cuidado é Fundamental Online, v. 8, n. 2, p.4197-211, 2016. Disponível em: https://www.ssoar.info/ssoar/handle/document/53764. Acesso em: 25 maio. 2020.

HALLAL, A. L. L. C. et al. The use of other tobacco products among Brazilian school children (PeNSE 2012). Cadernos de Saúde Pública, v. 33, n. 3, p.1-8, 2017. Disponível em: http://www.scielo.br/pdf/csp/v33s3/1678-4464-csp-33-s3-e00137215.pdf. Acesso em: 25 maio. 2020.

MACEDO, E. O. S.; CONCEICAO, M. I. G. Significações sobre adolescência e saúde entre participantes de um grupo educativo de adolescentes. Psicologia: Ciência e Profissão, v. 35, n. 4, p. 1059-73, 2015. Disponível em: http://www.scielo.br/pdf/pcp/v35n4/1982-3703-pcp-354-1059.pdf. Acesso em: 25 maio. 2020.

MALTA, D. C. et al. Trend of the risk and protective factors of chronic diseases in adolescents, National Adolescent School-based Health Survey (PeNSE 2009 e 2012). Revista Brasileira de Epidemiologia, v. 17, n. 1, p.77-91, 2014. Disponível em: http://www.scielo.br/pdf/rbepid/v17s1/1415-790X-rbepid-17-s1-00077.pdf. Acesso em: 25 maio. 2020.

OLIVEIRA-CAMPOS, M. et al. Risk and protection factors for chronic noncommunicable diseases in adolescentes in Brazilian capitals. Revista Brasileira de Epidemiologia, v. 21, n. 1, e180002, 2018. Disponível em: https://www.scielo.br/pdf/rbepid/v21s1/en_1980-5497-rbepid21-s1-e180002.pdf. Acesso em: 25 maio. 2020

PEIRSON, L. et al. Interventions for prevention and treatment of tobacco smoking in schoolaged children and adolescents: a systematic review and meta-analysis. Preventive Medicine, v. 85, p.20-31. Disponível em: https://www.ncbi.nlm.nih.gov/pubmed/26743631. Acesso em: 25 maio. 2020.

PEREIRA, K. A. S. et al. Risk and protective factors for noncommunicable diseases among adolescents. Revista Brasileira em Promoção da Saúde, v. 30, n. 2, p. 205-12, 2017. Disponível em: http://www.redalyc.org/pdf/408/40851821008.pdf. Acesso em: 25 maio. 2020.

THIOLLENT, M. Metodologia da pesquisa-ação. 17ª ed. São Paulo: Cortez; 2009. 
VALLADARES-GARRIDO, M. J. et al. Socioeducational factors associated with tobacco use in secondary education students in a rural area of northern Peru. Pediatría Atención Primaria, v. 19, n. 74, p.53-8, 2017. Disponível em: http://scielo.isciii.es/pdf/pap/v19n74/en_1139-7632pap-19-74-00e53.pdf. Acesso em: 25 maio. 2020.

VIERO, V. S. F. et al. Health education with adolescents: analysis of knowledge acquisition on health topics. Escola Anna Nery - Revista de Enfermagem, v.19, n. 3, p.484-90, 2015. Disponível em: http://www.scielo.br/pdf/ean/v19n3/en_1414-8145-ean-19-03-0484.pdf. Acesso em: 25 maio. 2020.

WORLD HEALTH ORGANIZATION. WHO global report on trends in prevalence of tobacco smoking. Geneva, 2015. Disponível em: https://www.who.int/tobacco/publications/surveillance/reportontrendstobaccosmoking/en/. Acesso em: 25 maio. 2020.

Recebido em: 21/09/2019

Aceito em: 19/06/2020 